\title{
A quick and simple spectrophotometric method to determine total carbon concentrations in root exudation samples
}

Eva Oburger ( $\sim$ eva.oburger@boku.ac.at )

Institute of Soil Research, University of Natural Resources and Life Sciences https://orcid.org/00000003-3213-2588

\section{Walter Wenzel}

Institute of Soil Research, University of Natural Resources and Life Sciences

\section{Michael Santangeli}

Institute of Analytical chemistry, University of Natural Resources and Life Sciences

\section{Research Article}

Keywords: Rhizodeposition, metabolomics, Root exudate, Dissolved Organic Carbon, Carbon dynamics, Rhizosphere

Posted Date: November 8th, 2021

DOI: https://doi.org/10.21203/rs.3.rs-1043445/v1

License: (9) This work is licensed under a Creative Commons Attribution 4.0 International License. Read Full License 


\section{Abstract}

\section{Purpose}

Root exudates are key components driving belowground interaction between plant, microbes and soil. While high-end analytical approaches provide advanced insights into exudate metabolite diversity, the amount of total carbon (C) released by roots is the most basic parameter that should always be measured when characterizing root exudation as it (i) provides quantitative information of $\mathrm{C}$ exuded into the surrounding soil and (ii) allows to relate the abundance of individual exudate compounds to total $\mathrm{C}$ released. Here we propose a simple and quick, spectrophotometry-based method to quantify total dissolved organic carbon (DOC) concentration in exudation samples that is based on measuring the absorption of a pre-filtered but otherwise untreated exudate sample at $260 \mathrm{~nm}$.

Method

Exudate samples collected from different species and experimental approaches were analysed with our DOC assay and results were compared with $\mathrm{C}$ concentrations obtained by liquid TOC-analyser, demonstrating the suitability of our method.

Conclusion

Our method will facilitate quick and inexpensive measurements and thus increase our understanding of root exudation and related rhizosphere processes.

\section{Introduction}

Roots release a large diversity of soluble or volatile organic molecules (i.e., root exudates) as well cell debris and sloughed-off root cap cells as they forage for water and nutrients. The functional importance of root exudates in driving belowground interactions between plant roots, soil microbes and soil matrix has been increasingly acknowledged in the last decades (e.g. Badri and Vivanco 2009; Coskun et al. 2017; Dennis et al. 2010; Sasse et al. 2018). Despite our awareness about the central role of exudates in rhizosphere processes, studies investigating exudate quality and quantity under natural growth conditions are still relatively scarce. The difficult choice of a suitable but also applicable exudate sampling procedure combined with challenging analytical approaches makes it easier to discuss the importance of exudates in soil than to actually investigate them (Oburger and Jones 2018). Most studies investigating root exudation in the past focused on individual compounds or compound classes (e.g. organic acids, amino acids, etc.), ignoring a large fraction of the total carbon (C) released by roots. Thanks to recent developments not only in analytical instrumentation, but also in computer power, available data processing software as well as metabolite data bases, the number of non-targeted metabolomic exudation studies aiming to reveal the entire metabolite composition released by roots has significantly increased in the past five years. While a more detailed understanding of exudate compound diversity will allow us to increasingly decipher the cross-talk between plants and microbes, it is important 
not to overlook the overall quantitative amount of carbon (C) released by roots. When focusing on specific exudate compounds using targeted analysis only, we miss the quantitative relevance of our target analytes compared to all other compounds released. Also, non-targeted metabolomics usually only

allows the identification of a fraction of all metabolites released as available data bases are still limited, and non-targeted metabolomic analysis only provides qualitative information, or semi-quantitative at best. Logically, we focus on the identified compounds when interpreting and discussing our results. It is therefore of crucial importance to relate compound specific results to total $\mathrm{C}$ exudation to consider the general trend of $C$ release in our data interpretation. Total $C$ release rates are known to differ between species when grown under the same conditions (Canarini et al. 2016) but also within one genotype when grown on different soils/substrates (Oburger et al. 2014; Sasse et al. 2020). Furthermore, total C exudation rates (per unit root biomass or root surface area) has been found to decrease with increasing plant development (Santangeli et al. in prep.) These findings highlight the importance to measure total C exudation in addition to individual metabolite composition. From an ecological perspective, information of changes in total $\mathrm{C}$ release rates under changing environmental conditions is highly valuable, even if compound specific information is missing or available for only a few metabolites.

Here we propose a simple and quick, photometry-based method to quantify total dissolved organic carbon (DOC) concentrations in exudation samples. Our method was applied to liquid exudation samples, hence targeting the soluble fraction of exudates released. Based on a previous study applying photometry to estimate DOC concentrations in soil solutions, stem flow, throughfall and surface waters at $254 \mathrm{~nm}$ (Brandstetter et al. 1996), we tested the applicability of this approach to exudate samples. Our DOC assay was performed using a plate reader suitable for 96 microwell plates and calibrated using an internal calibration with potassium phthalate including independent quality controls on each individual plate with certified reference material traceable to standard reference material from NIST. Wavelength scans allowed us to optimize our assay by selecting a wavelength that produced reliable results for total dissolved carbon concentrations in root exudation samples. To verify the suitability and reliability of our proposed photometry-based approach, we compared photometric results with results from DOC analysis by a liquid $\mathrm{C}$ analyser.

\section{Materials And Methods}

Method development

\section{Exudate samples}

In total, 68 exudation samples from different experiments including their respective blanks and 2 quality controls ( $1 \mathrm{mg} \mathrm{C} \mathrm{L}^{-1}$ and $5 \mathrm{mg} \mathrm{C} \mathrm{L}^{-1}$ ) (TOC Standard Solution 132253, Supelco, Germany) were used for method development. All exudation samples tested were collected using either a hydroponic-only or a soil-hydroponic hybrid approach. While plants were grown in an artificial nutrient solution in the first experimental approach, the latter combined soil growth with careful root washing followed by a short hydroponic sampling period (Oburger and Jones 2018). Experimental plants included the Zea mays root 
hair defective mutant rth3 and the corresponding wild-type siblings (Wen and Schnable 1994). Plants were grown on different experimental soils (soil-hydroponic hybrid approach) for different time periods prior to exudation sampling, either in the greenhouse, in growth cabinets or in the field. Exudation

sampling periods ranged between 2 and 4 hours. All exudates were sampled in HQ containing $0.01 \mathrm{~g} \mathrm{~L}^{-1}$ Micropur (Katadyn) to inhibit microbial decomposition of exuded metabolites from the sampling solution during the sampling process (Oburger et al. 2014). After the exudation sampling period, roots were removed from the sampling solution, samples were filtered using $0.2 \mu \mathrm{m}$ cellulose acetate filters (OE 66, Whatman, UK) and then stored at $-20^{\circ} \mathrm{C}$ to await analysis.

\section{Analysis of total dissolved organic carbon using a liquid TOC analyser}

A calibration for total dissolved organic carbon ranging from 0.5 to $40 \mathrm{mg} \mathrm{C} \mathrm{L}^{-1}$ was prepared using potassium phthalate (KHP, Elementar 35.00-0151) dissolved in HQ water. Aliquots (5 ml) of standards, blanks and exudation samples were analysed for total dissolved organic $\mathrm{C}$ by a Vario Elementar TOC analyser for liquid samples. Determination of $\mathrm{C}$ concentrations in samples was based on a high temperature catalytic oxidation approach, including the combustion of samples at $850^{\circ} \mathrm{C}$ followed by the determination of $\mathrm{CO}_{2}$ by an infrared $\mathrm{CO}_{2}$ detector. Prior to analysis, $40 \mu \mathrm{l}$ of $10 \% \mathrm{HCl}$ were added to each sample to ensure degassing of inorganic carbonates from the solution. However, tests revealed that in contrast to soil solution samples, inorganic carbonates are negligible in exudation samples (data not shown). Obtained results were then used to optimize and verify the suitability of our DOC photometric assay.

\section{Testing photometric absorption to determine total dissolved organic carbon concentrations in exudate samples}

A calibration for total dissolved organic carbon ranging from 0.5 to $40 \mathrm{mg} \mathrm{C} \mathrm{L}^{-1}$ was prepared using potassium phthalate (KHP, Elementar 35.00-0151) dissolved in HQ water. Calibration blanks, calibration standards as well as thawed exudation samples and sample blanks $(250 \mu \mathrm{l})$ were pipetted into 96 microwell plate (Greiner UV-STAR® flat-bottom) suitable for absorbance analysis in the UV/VIS spectrum. Next, these plates were scanned using a Tecan Infinite 200 PRO (Tecan, Switzerland). For method development and optimization, a wavelength scan from $230 \mathrm{~nm}$ to $370 \mathrm{~nm}$ was applied to all samples, blanks, and calibration standards. Obtained results where then compared against results obtained by the liquid DOC analyser. Regression plots were prepared and related statistical parameters were determined using Graphpad Prism 8.0.2 for Windows (GraphPad Sofware, San Diego, CA, USA) and $\mathrm{R}^{2}$ was calculated to evaluate the goodness of fit of the linear regression model.

\section{Final photometric assay to determine total dissolved organic carbon concentrations in exudate samples}


Prepare a calibration for total dissolved organic carbon ranging from 0 to $40 \mathrm{mg} \mathrm{CL}^{-1}$ using potassium phthalate (KHP, Elementar 35.00-0151) dissolved in HQ water as well as two independent quality control solutions (TOC Standard Solution 132253, Supelco, Germany). Pipette calibration blanks, standards, quality controls as well as exudate samples and respective sample blanks (250 $\mu$ l) into 96 well plate (e.g. Greiner UV-STAR® flat-bottom) suitable for absorbance analysis in the UV/VIS spectrum and measure absorbance at $260 \mathrm{~nm}$.

\section{Results}

\section{Identifying a suitable wavelength for photometric determination of total $\mathrm{C}$ concentrations in root exudate samples}

A comparison of wavelength scans of a calibration standard solutions $\left(10.1 \mathrm{mg} \mathrm{L}^{-1}\right)$ and an exudation sample of similar concentration $\left(10.3 \mathrm{mg} \mathrm{L}^{-1}\right)$ revealed differences in absorption behaviour across the investigated wavelength spectrum (230-370 nm, Figure 1a) but showed a good fit at wavelengths 258$264 \mathrm{~nm}$ (Figure 1b - highlighted in gey: keep in mind that the concentration in exudate sample is slightly higher than the $\mathrm{C}$ concentration in the standard. Our results further indicated that at $254 \mathrm{~nm}$ (i.e. the wavelength previously applied in a method published for determining DOC concentrations in soil solutions) $\mathrm{C}$ concentrations in exudate solutions are underestimated.

Figure 1 (a) Absorbance scans across the wavelength spectrum from 230-370 nm for one exemplary exudate sample (10.3 mg L-1) and one calibration standard (10.1 mg L-1). Red line indicates the selected wavelength $(260 \mathrm{~nm})$ for photometric DOC determination in exudation samples. (b) Absorbance scans across the wavelength spectrum from $250-280 \mathrm{~nm}$. Grey area indicates most suitable wavelength spectrum $(258-264 \mathrm{~nm})$.

This was confirmed by plotting the absorbance at $254 \mathrm{~nm}$ of all tested exudate samples and the included standard solutions versus the respective DOC concentrations separately determined by the liquid TOC analyser (Figure 2a). Table 1 shows that when measured at $254 \mathrm{~nm}$, the slope of the calibration standards (0.0121) deviated from the slope obtained by the exudation samples (0.0084). In contrast, plotting the same data using the absorbance at $260 \mathrm{~nm}$ resulted in the most comparable slopes for exudate samples (0.0065) and calibration standards (0.007) across all tested wavelengths (Figure $2 \mathrm{~b}$, Table 1, results from other wavelengths tested are not shown). Despite the differences in obtained slopes by both wavelengths, the $\mathrm{R}^{2}$ of the absorbance regressions were similar for exudation samples $(0.97)$ as well as for calibration standards (0.99), highlighting the robustness of this photometric assay.

Figure 2 (a) Regression plot of absorbance measured in exudate samples and calibration standards at $254 \mathrm{~nm}$ versus DOC concentrations determined separately by a liquid TOC analyser. (b) Regression plot 
of absorbance measured in exudate samples and calibration standards at $260 \mathrm{~nm}$ versus DOC concentrations determined separately by a liquid TOC analyser.

Table 1

Results from the linear regression models of absorbances of exudate samples plotted against the mg $\mathrm{L}^{-1}$ C measured with the liquid TOC analyser and calibration standards plotted against the known standard concentration, with absorbance of exudate samples and standards measured at 254 and $260 \mathrm{~nm}$.

\begin{tabular}{|c|c|c|c|c|}
\hline & Abs $\mathrm{Ex}_{260}$ vs TOC & $\begin{array}{l}\text { Abs } S_{t d} 260 \text { vs } \\
\text { Std } \\
\text { known }\end{array}$ & Abs $\mathrm{Ex}_{254}$ vs TOC & $\begin{array}{l}\text { Abs Sdt } \text { St54 }_{\text {vs }} \\
\text { Std }_{\text {known }}\end{array}$ \\
\hline & \multicolumn{4}{|c|}{ Best-fit values Best-fit values } \\
\hline Slope & 0.0065 & 0.0070 & 0.0084 & 0.0121 \\
\hline Y-intercept & 0.0507 & 0.0521 & 0.0554 & 0.0602 \\
\hline \multirow[t]{2}{*}{ 1/slope } & 153.9 & 143.5 & 119.7 & 82.53 \\
\hline & \multicolumn{4}{|l|}{ Std. Error } \\
\hline Slope & $1.41 \mathrm{E}-04$ & 1.98E-05 & 1.90E-04 & 4.32E-05 \\
\hline \multirow[t]{2}{*}{ Y-intercept } & $1.35 \mathrm{E}-03$ & 2.69E-04 & $1.82 \mathrm{E}-03$ & $5.86 \mathrm{E}-04$ \\
\hline & \multicolumn{4}{|c|}{ 95\% Confidence Intervals } \\
\hline Slope & 0.0062 to 0.0068 & 0.0069 to 0.0070 & 0.0080 to 0.0087 & 0.0120 to 0.0122 \\
\hline \multirow[t]{2}{*}{ Y-intercept } & 0.0480 to 0.0534 & 0.0515 to 0.0528 & 0.0517 to 0.0590 & 0.0588 to 0.0616 \\
\hline & \multicolumn{4}{|l|}{ Goodness of Fit } \\
\hline $\mathrm{R}^{2}$ & 0.9698 & 0.9999 & 0.9668 & 0.9999 \\
\hline Equation & $\begin{array}{l}Y=0.0065 * X+ \\
0.0507\end{array}$ & $\begin{array}{l}Y=0.007 * X+ \\
0.0521\end{array}$ & $\begin{array}{l}Y=0.0084 * X+ \\
0.0554\end{array}$ & $\begin{array}{l}Y=0.0121 * X+ \\
0.0602\end{array}$ \\
\hline $\begin{array}{l}\text { Nr. of } X \\
\text { values }\end{array}$ & 68 & 9 & 68 & 9 \\
\hline
\end{tabular}

In addition, we used the respective calibration equations obtained for both wavelengths ( $254 \mathrm{~nm} \& 260$ $\mathrm{nm}$ ) to calculate DOC concentrations in our exudate samples and then plotted them against the concentrations separately determined by a liquid TOC analyser (Figure 3). Figure 3 and Table 2 shows that using $254 \mathrm{~nm}$ leads to an underestimation of $\mathrm{C}$ concentrations in our exudation samples, with a $1 /$ slope ratio of 1.402 . Plotting results obtained at $260 \mathrm{~nm}$ shows against concentrations determined by the TOC analyser resulted in a 1/slope ratio close to 1 (1.034) (Table 2). This further supports the greater suitability of using $260 \mathrm{~nm}$ instead of $254 \mathrm{~nm}$ to reliable determine dissolved organic carbon concentrations in exudate samples. 
Table 2

Results from the linear regression model of DOC measured at 260 and $254 \mathrm{~nm}$ versus the $\mathrm{mg} \mathrm{L}^{-1} \mathrm{C}$ measured with the liquid TOC analyser.

\begin{tabular}{|lll|}
\hline & DOC $_{260}$ vs DOC & DOC $_{254}$ Vs DOC $_{\text {TOC }}$ \\
\hline & Best-fit values & \\
\hline Slope & 0.9671 & 0.7133 \\
\hline Y-intercept & 0.2708 & -0.0996 \\
\hline 1/slope & 1.034 & 1.402 \\
\hline Slope & Std. Error & 0.0168 \\
\hline Y-intercept & 0.0267 & 0.1613 \\
\hline Slope & 0.2555 & $95 \%$ Confidence Intervals \\
\hline Y-intercept & 0.9138 to 1.020 & 0.6797 to 0.7469 \\
\hline & -0.2393 to 0.7810 & -0.4216 to 0.2224 \\
\hline R square & Goodness of Fit & \\
\hline Equation & 0.9521 & 0.9645 \\
\hline Nr. of X values & 68 & $Y=0.7133^{*} X-0.09960$ \\
\hline
\end{tabular}

\section{Discussion And Outlook}

Here we tested and validated a quick and simple method to determine DOC concentrations in exudate samples by simple means of using a plate photometer in combination with 96-microwell plates suitable for UV/VIS light spectrum. Our tests revealed that measuring the absorption of pre-filtered $(0.2 \mu \mathrm{m})$ but otherwise unprocessed exudate samples at $260 \mathrm{~nm}$ is an accurate and fast approach to quantify total C (DOC) concentrations in exudate solutions. A previous study proposed $254 \mathrm{~nm}$ as the most suitable wavelength to determine DOC concentrations in soil solutions (Brandstetter et al. 1996). In contrast to this study, Brandstetter et al. (1996) did not include separate, independent calibration and reference standards in their photometric analysis but simply correlated results obtained by a TOC analyser with the absorbance measured at $254 \mathrm{~nm}$. Correlating results from a TOC analyser with photometric absorption at $254 \mathrm{~nm}$ and $260 \mathrm{~nm}$ in our study revealed equally good fits for both wavelength $\left(R^{2}=0.96\right.$ and 0.95 respectively, Table 2). However, including independent calibration standards (Figure 2a,b) and plotting results calibrated with the obtained calibration curves measured at 254 and $260 \mathrm{~nm}$ versus results from the TOC-analyzer (Figure 3), showed that using $254 \mathrm{~nm}$ to determine total $\mathrm{C}$ in exudate samples underestimates $\mathrm{C}$ concentrations. Including independent calibrations standards and quality controls 
together with samples on each plate subjected to analysis by the plate photometer ensures not only accurate calibration of DOC concentrations but also allows to account for instrument specific variation as well as daily variation of instrument performance. Our approach is therefore more robust compared to published regression equations that might be affected by instrument performance. Whether or not our proposed method is also suitable for DOC analysis in soil solutions and potentially also superior to absorption measured at $254 \mathrm{~nm}$ as proposed by Brandstetter et al. (1996) remains to be tested as this was out of scope in this study.

Compound specific as well as non-targeted analytical approaches to capture species- and treatmentspecific root exudation often require high end analytical equipment and time-consuming method development. Even though they deliver highly relevant in-depth information on exudation patterns, obtained results miss the quantitative relation of individual compounds, compound classes or metabolite fingerprints to total carbon exuded if DOC concentrations in exudate samples are not determined. Our proposed photometric assay holds promise to become a universally applicable standard method in exudate analysis as it enables experimentalists to accurately determine DOC concentrations in exudate samples with minimum effort using standard laboratory equipment (UV-photometer). From an ecological point of view, quantitative information on total $\mathrm{C}$ exuded per plant if total root biomass is presented (mg or $\mu \mathrm{mol} \mathrm{C}$ plant $^{-1} \mathrm{~h}^{-1}$ ), or per unit root biomass or root surface area ( $\mathrm{mg}$ or $\mu \mathrm{mol} \mathrm{C} \mathrm{g} \mathrm{root} \mathrm{dry} \mathrm{weight}{ }^{-1}$ $\mathrm{h}^{-1} / \mathrm{mg}$ or $\mu \mathrm{mol} \mathrm{Cg}$ root surface area ${ }^{-1} \mathrm{~h}^{-1}$ ) is highly relevant as roots and exudation-fuelled rhizosphere processes are known to play a central role in soil carbon and nutrient cycling. Furthermore, the choice of exudation sampling approach and accurate data presentation is critical for obtaining ecologically relevant results that allow comparison between different studies (Oburger and Jones 2018). Increasing the number of data sets reporting $\mathrm{C}$ exudation rates by different plant species under different environmental conditions will not only increase our understanding about $\mathrm{C}$ exudation dynamics but will also improve the accuracy of model predictions of $\mathrm{C}$ dynamics in soils and ecosystems.

\section{Declarations}

\section{Acknowledgements}

This work was conducted within the framework of the priority program 2089, funded by the Deutsche Forschungsgemeinschaft (DFG, German Research Foundation) - Project numbers: 403803214 and the ERC Starting Grant 801954 PhytoTrace. Seeds of the maize wild-type and mutant $r$ th 3 were provided by Caroline Marcon and Frank Hochholdinger (University of Bonn). We thank Lisa Stein for helping with photometric measurements.

\section{References}

Badri DV, Vivanco JM (2009) Regulation and function of root exudates. Plant Cell and Environment 32: 666-681. doi: 10.1111/j.1365-3040.2009.01926.x. 
Brandstetter A, Sletten RS, Mentler A, Wenzel WW (1996) Estimating dissolved organic carbon in natural waters by UV absorbance (254 nm). Zeitschrift Fur Pflanzenernahrung Und Bodenkunde 159: 605-607.

Canarini A, Merchant A, Dijkstra FA (2016) Drought effects on Helianthus annuus and Glycine max metabolites: from phloem to root exudates. Rhizosphere 2: 85-97. doi:

https://doi.org/10.1016/j.rhisph.2016.06.003.

Coskun D, Britto DT, Shi WM, Kronzucker HJ (2017) How Plant Root Exudates Shape the Nitrogen Cycle. Trends in Plant Science 22: 661-673. doi: 10.1016/j.tplants.2017.05.004.

Dennis PG, Miller AJ, Hirsch PR (2010) Are root exudates more important than other sources of rhizodeposits in structuring rhizosphere bacterial communities? FEMS microbiology ecology 72: 313-327. doi: 10.1111/j.1574-6941.2010.00860.x.

Oburger E, Gruber B, Schindlegger Y, Schenkeveld WDC, Hann S, Kraemer SM, Wenzel WW, Puschenreiter M (2014) Root exudation of phytosiderophores from soil-grown wheat. New Phytologist 203: 1161-1174. doi: $10.1111 / \mathrm{nph} .12868$.

Oburger E, Jones DL (2018) Sampling root exudates - Mission impossible? Rhizosphere 6: 116-133. doi: https://doi.org/10.1016/j.rhisph.2018.06.004.

Sasse J, Kosina SM, de Raad M, Jordan JS, Whiting K, Zhalnina K, Northen TR (2020) Root morphology and exudate availability are shaped by particle size and chemistry in Brachypodium distachyon. Plant Direct 4: 14. doi: 10.1002/pld3.207.

Sasse J, Martinoia E, Northen T (2018) Feed Your Friends: Do Plant Exudates Shape the Root Microbiome? Trends in Plant Science 23: 25-41. doi: 10.1016/j.tplants.2017.09.003.

Wen T-J, Schnable PS (1994) Analyses of mutants of three genes that influence root hair development in Zea mays (Gramineae) suggest that root hairs are dispensable. American Journal of Botany 81: 833-842. doi: https://doi.org/10.1002/j.1537-2197.1994.tb15564.x.

\section{Figures}



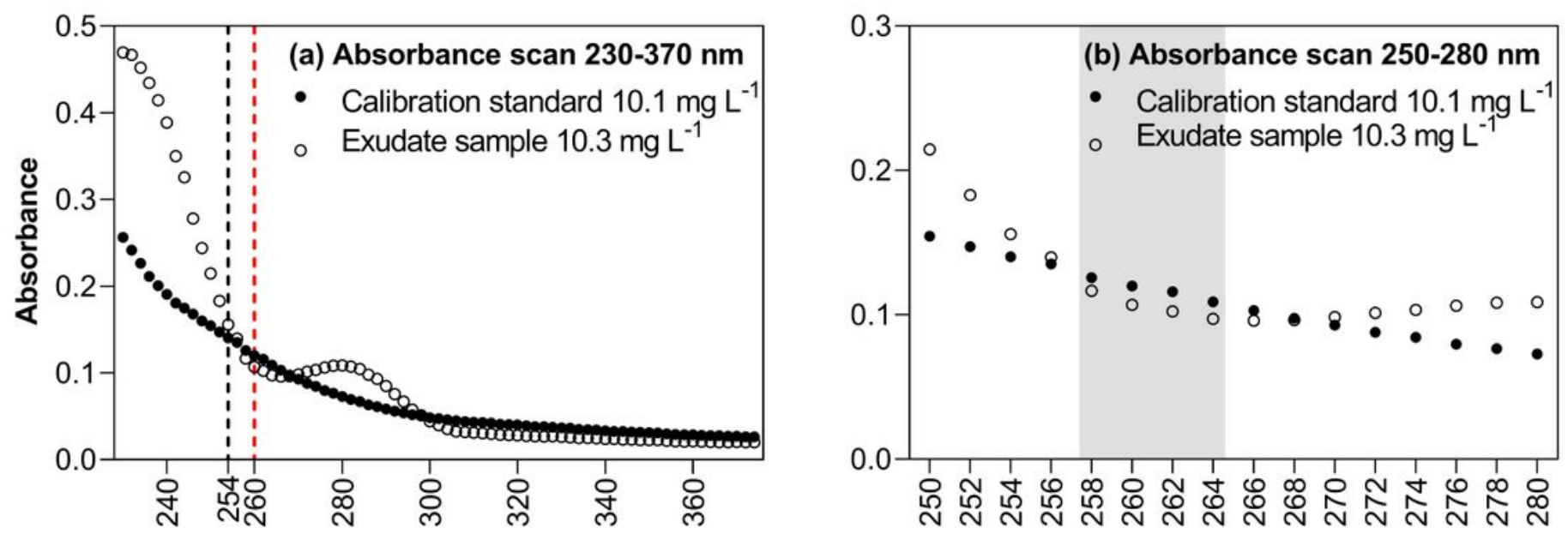

Wavelength $(\mathrm{nm})$

\section{Figure 1}

(a) Absorbance scans across the wavelength spectrum from 230-370 nm for one exemplary exudate sample (10.3 mg L-1) and one calibration standard (10.1 mg L-1). Red line indicates the selected wavelength $(260 \mathrm{~nm})$ for photometric DOC determination in exudation samples. (b) Absorbance scans across the wavelength spectrum from 250-280 nm. Grey area indicates most suitable wavelength spectrum (258-264 nm).

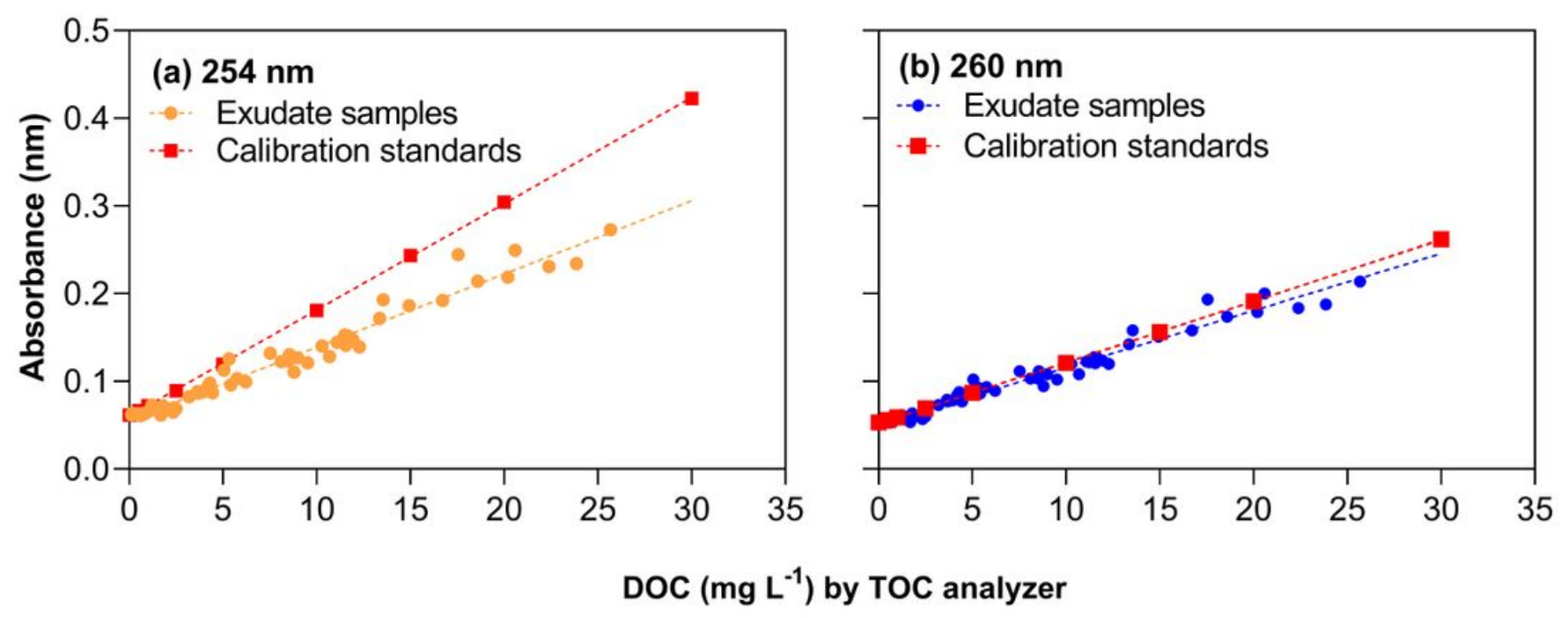

Figure 2

(a) Regression plot of absorbance measured in exudate samples and calibration standards at $254 \mathrm{~nm}$ versus DOC concentrations determined separately by a liquid TOC analyser. (b) Regression plot of absorbance measured in exudate samples and calibration standards at $260 \mathrm{~nm}$ versus DOC concentrations determined separately by a liquid TOC analyser. 


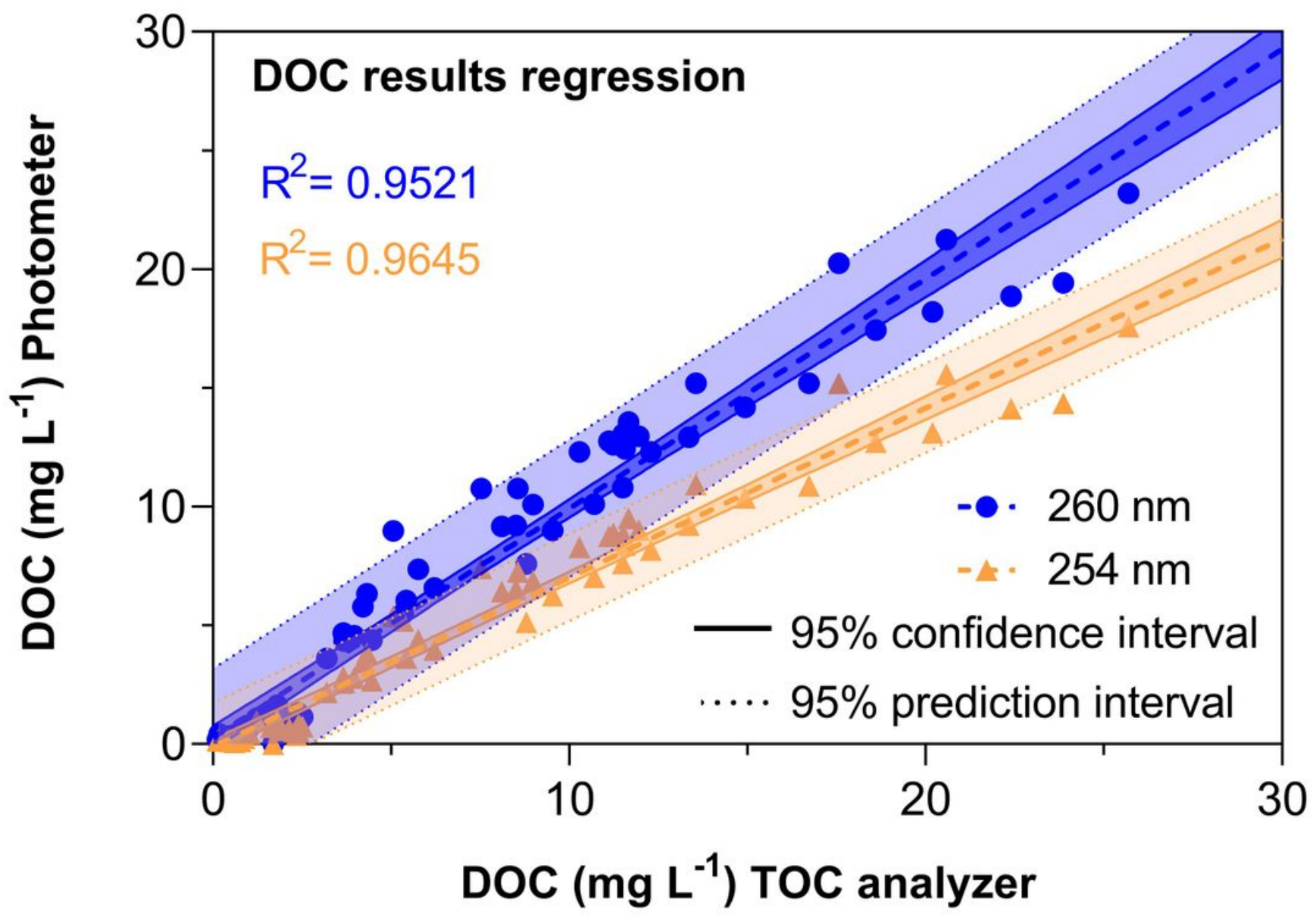

Figure 3

Regression plot of DOC concentrations of exudate samples calibrated based on wavelength specific absorbance $(254 \mathrm{~nm}, 260 \mathrm{~nm})$ and determined by a liquid TOC analyser. Coloured areas within dotted lines represent the $95 \%$ prediction interval, while area within the continuous lines represent the $95 \%$ confidence interval. 